\title{
Revisión de estudios e investigaciones relacionadas con la comorbilidad diagnóstica de los Trastornos del Espectro del Autismo de Alto Funcionamiento (TEA-AF) y los trastornos de ansiedad
}

\author{
Myriam De-la-Iglesia y José-Sixto Olivar
}

Universidad de Valladolid (España)

\begin{abstract}
Resumen: El objeto del estudio es revisar las investigaciones sobre la comorbilidad entre los Trastornos del Espectro del Autismo de Alto Funcionamiento (TEA-AF) y los trastornos de ansiedad con un triple propósito: a) analizar los problemas metodológicos de los estudios de investigación, b) plantear las hipótesis explicativas sobre la comorbilidad, con un posicionamiento y propuesta sobre las mismas teniendo en cuenta las nuevas propuestas del DSM-V (APA, 2011) y c) proponer líneas futuras de investigación. Se concluye que hay una necesidad urgente de desarrollar estrategias e instrumentos específicos que ayuden a la evaluación diferencial de la sintomatología comórbida y que favorezcan propuestas de tratamientos más eficaces. Se destaca la llamativa escasez de estudios de investigación sobre este tema en países de lengua hispana.

Palabras clave: Trastorno del Espectro del Autismo; Autismo de alto funcionamiento; Trastorno de Asperger; Comorbilidad; Ansiedad.
\end{abstract}

Title: Revision of studies and researches related with diagnostic comorbidity of Autism Spectrum Disorder-High Functioning and anxiety disorders. Abstract: The aim of this study is to review the state of the art of research on comorbidity of Autism Spectrum Disorder-High Functioning (ASD$\mathrm{AF}$ ) and anxiety disorders with a threefold purpose: a) to analyse the methodological problems of research studies, b) to present hypothesis on comorbidity, adopting an approach taking into account the new proposal of DSM-V (APA, 2011), and c) to suggest future research lines. Our study concludes that there is an urgent need to develop specific strategies and instruments that help the differential assessment of comorbidity symptomatology and that favour proposals of more efficient treatments. An outstanding lack of research studies on this topic in spanish-speaking countries is highlighted.

Key words: Autism Spectrum Disorder; High Functioning Autism; Asperger's Disorder; Comorbidity; Anxiety.

\section{Introducción}

Actualmente existe un consenso generalizado sobre la necesidad de una mayor profundización en el diagnóstico diferencial y la comorbilidad de los Trastornos del Espectro del Autismo (en adelante, TEA). Los criterios diagnósticos de este grupo se recogen hasta el momento en el DSM-IV-TR como Trastornos Generalizados del Desarrollo (TGD, Asociación Americana de Psiquiatría -en adelante, APA-, 2002), que incluyen el Trastorno Autista, el Trastorno Desintegrativo Infantil, el Síndrome de Rett, el Trastorno Generalizado del Desarrollo No especificado y el Trastorno de Asperger (en adelante, TA). Estos trastornos comparten lo que se ha venido en denominar "la tríada de Wing" desde la perspectiva del continuo o dimensión (deficiencias en la relación social, deficiencias en la comunicación social y deficiencias en la simbolización, Wing y Gould, 1979).

Sin embargo, los expertos internacionales para la elaboración de los futuros CIE 11 y DSM-V plantean cambios sustanciales en la categoría TGD (Organización Mundial de la Salud, 2011; APA, 2011). En concreto, proponen que el Trastorno autista se convierta en el único diagnóstico posible de la actual categoría TGD y pase a llamarse TEA, especificando los niveles de afectación. Este cambio de denominación trata de enfatizar la visión dimensional del trastorno en las diferentes áreas afectadas y la dificultad de establecer límites precisos entre los subgrupos dentro de la misma categoría. En cuanto al TA, proponen que se elimine como ca-

Dirección para correspondencia [Correspondence address]: Myriam De-la-Iglesia Gutiérrez. Facultad de Educación y Trabajo Social. Universidad de Valladolid. Departamento de Psicología. Campus Miguel Delibes. Paseo de Belén, 1. 47011-Valladolid (España).

E-mail: mdelaig@psi.uva.es

Miembros Asociados del Laboratoire LPPS, EA 405 (France). tegoría independiente, siendo subsumida en la de TEA, pues la investigación no avala que sea distinto del autismo de alto nivel de funcionamiento cognoscitivo.

$\mathrm{La}$ comorbilidad se refiere al solapamiento o coocurrencia de síntomas pertenecientes a distintos trastornos en una misma persona. Varias son las posibles explicaciones de la comorbilidad. Caron y Rutter (1991), sugieren que la co-ocurrencia de dos o más trastornos (1) puede ser el resultado del solapamiento de factores de riesgo; o (2) el resultado de una sola condición con múltiples manifestaciones; o (3) que un trastorno cause o disminuya el umbral para la expresión de otro; o (4) un trastorno represente una manifestación temprana de otro; o (5) la comorbilidad puede ser debida al solapamiento de criterios diagnósticos.

Las investigaciones empíricas sobre comorbilidad en los TEA son escasas, especialmente si se comparan con las generadas en el campo de los trastornos de inicio en la infancia y la adolescencia en general o de la discapacidad intelectual en particular. Además, sería necesario un estudio específico de la sintomatología comórbida en este colectivo, pues puede mostrar características diferentes que en la población general (Matson y Nebel-Schwalm, 2007).

La co-ocurrencia del autismo con otros trastornos es un hecho ampliamente constatado (Ghaziuddin, Ghaziuddin y Greden, 2002). Así, se llega a concluir que más que una excepción, "la comorbilidad es la regla común en el autismo y, aunque menos documentada, en el TA" (Gillberg y Billstedt, 2000, p. 327). Autores como Rutter, Bailey, Bolton, y Le Couteur informan en 1994 de que esto es así en al menos el 10\% de los casos. Estudios más recientes señalan que la comorbilidad psicopatológica en los TEA se produce en el 65\%-80\% de los casos (de Bruin, Ferdinand, Meester, de Nijs, y Verheij, 2006; Leyfer et al., 2006). Estas tasas son significativamente 
mayores que en los grupos de personas con discapacidad intelectual sin autismo (Brereton, Tonge, y Einfeld, 2006).

Gillberg y Billstedt (2000) señalan además la posibilidad de que estas condiciones de comorbilidad puedan ser marcadores de una patofisiología subyacente compartida entre los TEA y dichos trastornos comórbidos, lo que puede abrir nuevas vías de investigación para el futuro.

En las personas con TEA de alto nivel de funcionamiento cognitivo (en adelante, TEA-AF) en ocasiones resulta complicado el diagnóstico diferencial (Damián y Hernández, 1997), especialmente con los trastornos del desarrollo del lenguaje de tipo mixto, como el "trastorno semánticopragmático" (Bishop, 1989; Volkmar y Klin, 2000) y los trastornos del espectro "esquizo", sobre todo con el "trastorno esquizoide de la personalidad" (Volkmar y Klin, 2000; Wolf, 2000). En este sentido, conviene recordar la alta tasa de comorbilidad existente en los Trastornos de Personalidad como resultado, en muchos casos, de los propios criterios utilizados para la realización del diagnóstico (Salavera, Puyuelo, Tricás y Lucha, 2010).

Tradicionalmente, los trastornos frecuentemente implicados en el diagnóstico diferencial del TA, son: el Trastorno de déficit de atención con hiperactividad (Gadow, DeVincent, Pomeroy y Azizian, 2004; Gillberg y Billstedt, 2000; Green, Gilchrist, Di Burton y Cox, 2000; Holguin, 2003; Klin y Volkmar, 1997; Lecavalier, 2006; Leyfer et al., 2006; Sverd, 2003; Sterling, Dawson, Estes, y Greenson, 2008; Volkmar y Klin, 2000); el Trastorno de Gilles de la Tourette (Gadow et al., 2004; Gillberg y Billstedt, 2000; Green et al., 2000; Lecavalier, 2006; Leyfer et al., 2006; Sverd, 2003; Sterling et al., 2008; Volkmar y Klin, 2000); el Trastorno obsesivo compulsivo, con una conducta y pensamiento rígidos e.g. perseveraciones, conducta ritualística- (Gillberg y Billstedt, 2000; Gillott, Furniss y Walter, 2001; Kim, Szatmari, Bryson, Streiner y Wilson, 2000; Lecavalier, 2006; Leyfer et al., 2006; Sverd, 2003; Sterling et al., 2008; Tantam, 2000; Volkmar y Klin, 2000); la manía, depresión y ansiedad - fobias específicas y ansiedad generalizada- (Gadow et al., 2004; Gillott et al., 2001; Green et al., 2000; Kim et al., 2000; Lecavalier, 2006; Leyfer et al., 2006; Sverd, 2003; Sterling et al., 2008; Tantam, 2000; Volkmar y Klin, 2000); la Esquizofrenia (Gillberg y Billstedt, 2000; Holguin, 2003; Volkmar y Klin, 2000; Wolf, 1998); la agresiones (Gadow et al., 2004; Gillberg y Billstedt, 2000; Holguin, 2003; Lecavalier, 2006; Leyfer et al., 2006; Sverd, 2003; Sterling et al., 2008; Volkmar y Klin, 2000; Wolf, 1998); y otros como la catatonia, los trastornos de la alimentación, el mutismo selectivo o los Trastornos de sueño (Gillberg y Billstedt, 2000).

En este trabajo presentamos el estado de conocimiento y el debate actual sobre la comorbilidad diagnóstica de los TEA-AF y los trastornos de ansiedad. Para ello, aportamos una revisión de los estudios e investigaciones científicas relacionadas con la comorbilidad diagnóstica en los TEA-AF con los trastornos de ansiedad. Se pretende revisar y analizar el conocimiento científico actual para determinar si esta comorbilidad puede ser debida a un solapamiento de criterios diagnósticos, o si los TEA-AF son una condición facilitadora, que disminuye el umbral para los trastornos de ansiedad (Caron y Rutter, 1991). Se propician y establecen posibles vías de investigación futuras. La escasez de trabajos descriptivos, epidemiológicos, etiológicos y de intervención en esta área en países de lengua hispana plantea además la necesidad de realizar estudios contextualizados. Todos estos estudios ayudarían al avance no sólo científico, sino a la mejora de la calidad de vida de los propios afectados y de sus cuidadores y familias, en las que conocemos que existen mayores tasas de estrés respecto de la población general (Seguí, Ortiz y De Diego, 2008).

\section{Objetivos del estudio}

Los propósitos de este trabajo se centran en: a) Exponer el estado actual del corpus científico sobre comorbilidad de los TEA y los trastornos de ansiedad, b) Analizar los posibles problemas metodológicos de las diferentes investigaciones en este ámbito de estudio, según su influencia en las dificultades halladas para el consenso en este campo, c) Analizar y organizar el corpus de estudios que presentan diferentes explicaciones sobre la comorbilidad (diagnóstica o sintomatológica), y elaborar y aportar una propuesta final reflexiva, acorde con los resultados obtenidos a partir de estos análisis.

\section{La ansiedad en los trastornos del espectro del autismo de alto funcionamiento}

Según distintos estudios de investigación, la depresión y la ansiedad son los trastornos psicopatológicos más comunes en personas con TEA (Ghaziuddin, 2002; Ghaziuddin et al., 2002; Gillott et al., 2001; Kim et al., 2000; White y Roberson-Nay, 2009; White, Oswald, Ollendick, y Scahill, 2009). Todo ello teniendo en cuenta las dificultades de diferenciación entre ambos cuadros de las que nos informa la literatura (para una revisión, ver Sanz, 1993).

En la actualidad se vienen considerando unas tasas de prevalencia de entre el $11 \%$ y el $84 \%$ de niños y jóvenes con TEA que experimentan cierto grado de ansiedad (White y Roberson-Nay, 2009; White et al., 2009). Esta gran variabilidad puede deberse a los diferentes métodos de evaluación de la sintomatología. En unos casos, la evaluación se lleva a cabo mediante cuestionarios (por ejemplo, Gillott et al., 2001), o instrumentos no validados para su uso en los TEA (Mazefsky, Folstein y Lainhart, 2008). Además, para poder saber el verdadero índice de comorbilidad sería necesario un mayor número de estudios epidemiológicos de gran escala, frente a los datos surgidos de muestras de las personas que son remitidas a los servicios psiquiátricos, y por tanto ya con indicios de la presencia de un malestar (White et al., 2009; Wing, 1981).

En su amplio estudio sobre comorbilidad diagnóstica de trastornos de ansiedad en los TEA, De Bruin et al. (2006) encontraron que, de los 94 sujetos de estudio, más del 55\% 
cumplía criterios clínicos para al menos un trastorno de ansiedad. Simonoff et al. (2008) informaron de una tasa global de diagnóstico de un trastorno de ansiedad de casi el $42 \%$ en su muestra de estudio de 112 personas con TEA, siendo la ansiedad social el más prevalente $(29 \%)$. Este tipo de ansiedad también es destacada por otros autores, como en el estudio de Bellini (2004), quien informa de que 20 jóvenes (casi la mitad de la muestra total, compuesta por 41 adolescentes con TEA) mostraban niveles clínicamente significativos de ansiedad social; o en el trabajo de Melfsen, Walitza y Warnke (2006), donde al evaluar los síntomas de fobia social y ansiedad en 341 jóvenes con trastornos psicopatológicos de 7 a 18 años (Trastorno de Asperger, $n=7$ ), el grado de ansiedad social en el grupo con TA puntúa ligeramente por encima del punto de corte clínico indicativo de la ansiedad social y es significativamente mayor que el del grupo control de niños y adolescentes sin trastornos $(n=1197)$.

Las dificultades de ansiedad están presentes en todos los niveles cognitivos del autismo, aunque el tipo de problema de ansiedad específico sí parece estar influenciado por la capacidad cognitiva (White et al., 2009). Sukhodolsky et al. (2008) hallaron que los niños con mayor cociente intelectual y mayor deterioro social mostraron síntomas más graves de ansiedad. En el mismo sentido, en el estudio de Weisbrot, Gadow, DeVincent y Pomeroy (2005), a partir de la información de inventarios de evaluación de comorbilidad diagnóstica de familiares y profesores, obtuvieron que los niños con TA del grupo de 6 a 12 años presentan mayores puntuaciones de ansiedad que los niños con Trastorno autista, especialmente en la sintomatología relacionada con el trastorno de ansiedad generalizada. En el grupo de menor edad (3 a 5 años), aunque la información de los familiares no destacaba una mayor sintomatología ansiógena respecto al grupo de padres con hijos con desarrollo típico, la información de los profesores sí revelaba más síntomas de ansiedad. Además, los niños con autismo de 6 a 12 años puntuaron significativamente más alto en síntomas psicóticos (por ejemplo, delirios, afecto inapropiado) en comparación con los niños con menores niveles de ansiedad. Por último, encuentran que aquellos niños con mayores niveles de ansiedad poseían un mayor funcionamiento cognitivo que el grupo de niños con autismo que puntuaron menos en ansiedad.

En comparación con la población general, la mayoría de los estudios sugieren que los niños con autismo muestran índices significativamente más elevados tanto en ansiedad como en depresión, si bien es destacable la variabilidad en los datos resultantes dependiendo de los estudios de referencia consultados. Así, se informa de unas tasas que incluyen un amplio abanico dependiendo de estudios, desde el $7 \%$ al $84 \%$ en ansiedad, y del $4.4 \%$ al $57.6 \%$ en depresión (por ejemplo, Brereton et al., 2006; Ghaziuddin et al., 2002; Gillott et al., 2001; Mazefsky et al., 2008; Melfsen et al., 2006; Stewart, Barnard, Pearson, Hasan y O'Brien, 2006).

Respecto a otros grupos diagnósticos, las tasas de comorbilidad con la ansiedad del grupo de personas con TEA también son estadísticamente significativas (White et al.,
2009). Por ejemplo, Green et al. (2000) realizaron un estudio mediante entrevistas semiestructuradas tanto a ambos padres como a los propios jóvenes para valorar la prevalencia de problemas emocionales y de conducta. La muestra estaba constituida por 20 niños con TA y 20 niños con trastorno de conducta (edad media= 13 años). Aunque se hallaron elevadas tasas de síntomas psicopatológicos en ambos grupos (depresión, ideación suicida y comportamientos desafiantes), los adolescentes con TA mostraron significativamente mayores síntomas de ansiedad. En concreto, en más de la mitad de los participantes (11 de 20) del grupo con TA se hallaron dos o más síntomas de ansiedad. Este grupo mostraba mayores dificultades en el funcionamiento psicosocial a pesar de su buen funcionamiento cognitivo. Por esta razón, argumentan que las dificultades sociales podrían estar haciéndoles más vulnerables a síntomas afectivos secundarios, similares a los que pueden aparecer en otras personas con dificultades en la socialización (por ejemplo, personas con trastornos de conducta). Ambos grupos manifestaron una toma de conciencia (insight) insuficiente sobre sus dificultades, aunque en dos tercios de las personas con TA existía cierto grado de conciencia. La explicación de estos problemas emocionales y afectivos (depresión y ansiedad) es que pueden estar reflejando una toma de conciencia de sus dificultades, mientras que la conducta antisocial estaría más relacionada con déficit primarios en empatía.

En la misma línea, Evans, Canavera, Kleinpeter, Maccubbin y Taga (2005) comparan los miedos y problemas de conducta de 25 niños con TEA con los de 43 niños con síndrome de Down y con los de dos grupos control con desarrollo típico: 45 niños equiparados en edad mental y 37 equiparados en edad cronológica. Encontraron que los niños con TEA tenían más miedos que cualquiera de los niños con Síndrome de Down, y destacan especialmente los temores específicos situacionales (e.g. espacios pequeños y situaciones médicas). Subrayan además entre sus conclusiones la relación entre los problemas de conducta de los niños con TEA y los índices de ansiedad, concluyendo que los problemas exteriorizados (por ejemplo, problemas de conducta, falta de atención) estaban más fuertemente relacionados con los temores en los sujetos con TEA que en los niños con Síndrome de Down y con desarrollo típico. A tenor de estos resultados, hipotetizan que los sujetos con TEA pueden expresar sus temores o la ansiedad a partir de problemas externalizados, o que simplemente estos problemas tienen más probabilidades de co-ocurrir en esta patología. Estos resultados, no obstante, tienen un alcance limitado, pues tanto la información diagnóstica categorial como la relativa a los problemas de ansiedad fueron proporcionadas exclusivamente por los familiares de los afectados, no habiendo sido contrastados estos datos por los investigadores. Además, la evaluación de los temores de los niños se realizó mediante escalas diseñadas "ad hoc" centradas en determinados miedos (e.g. entornos sanitarios, animales, etc).

En cuanto a los tipos más frecuentes de trastornos y sintomatología ansiógena en los TEA, los resultados de los es- 
tudios concluyen que en los niños son las fobias simples, trastornos de ansiedad generalizada, trastornos de ansiedad de separación, trastornos obsesivo-compulsivos y fobia social. En este sentido, resultan de interés los resultados del estudio de Orgilés, Espada, García, Méndez e Hidalgo (2011), pues en su muestra de 1407 niños españoles de edades entre los 8 y 12 años, al evaluar las diferencias en la ansiedad por separación en función del sexo y la edad, obtienen que esta ansiedad se muestra significativamente más presente en niñas que en niños, algo que contrasta con la preponderancia del género masculino en los TEA. En este sentido, también en población española sin trastornos, López, Alcántara, Fernández, Castro y López (2010) evaluaron la prevalencia de ansiedad en una muestra clínica de 300 niños y niñas de la misma cohorte de edad (8-12 años) y obtienen que son más varones los que muestran índices de ansiedad, no realizándose en este caso análisis diferenciales por tipos de ansiedad. Por otra parte, no se hallan datos destacables en población infantil con TEA en otros trastornos de ansiedad como por ejemplo la agorafobia, trastornos de estrés post-traumático, etc (White et al., 2009). Sin embargo, se destaca la controversia en el diagnóstico diferencial debido al solapamiento sustancial de la sintomatología ansiógena con la propia de los TEA y con la de la depresión -por ejemplo, las comprobaciones compulsivas y ritualísticas o la evitación social(Matson y Nebel-Schwalm, 2007; White et al., 2009). Así, no siempre está claro el diagnóstico de trastorno comórbido o diferencial al de TEA, lo que puede suponer una considerable variabilidad en los índices de prevalencia.

Kim et al. (2000) observaron mayores tasas de alteraciones del estado de ánimo (depresión) y de ansiedad estadísticamente significativas en los 59 niños con TEA-AF de entre 9 y 14 años que participaron en su estudio en comparación con el grupo control con desarrollo típico ( $n=1751$ niños). En concreto, 13.6\% mostraban ansiedad generalizada clínicamente significativa y $8.5 \%$ ansiedad de separación.

Gillott et al. (2001) exploran la naturaleza de la ansiedad en niños con TEA-AF. Para ello, evaluaron la ansiedad general y la preocupación social relacionada con la ansiedad social de niños con una media de edad de 10 años. La muestra estaba formada por 15 niños con TEA-AF y dos grupos control (15 niños con un desarrollo típico y 15 con alteraciones específicas del lenguaje). Sus resultados muestran que los niños con TEA-AF puntuaban más alto en ansiedad que los grupos de comparación en ambas medidas. Además, la mitad de los niños con TEA-AF puntuaron más alto que el punto de corte para trastornos de ansiedad. En concreto, las puntuaciones más altas se hallan en ansiedad de separación y trastorno obsesivo-compulsivo. Este grupo también mostró mayores índices de preocupaciones sociales que el resto de los grupos control. De los resultados de este estudio se concluye que los niños con TEA-AF pueden exhibir el espectro de preocupaciones sociales, tanto de manera encubierta (por ejemplo, pensando repetidamente sobre ellas) como abiertamente (por ejemplo, evitando activamente la interacción social con sus compañeros).
Russell y Sofronoff (2005) evaluaron la concordancia de la información sobre síntomas de ansiedad ofrecida por los padres y por los propios niños mediante escalas de ansiedad y cuestionarios sobre preocupación social. Además, compararon estos resultados con los de un grupo de niños con fobia social y otro con desarrollo típico. La muestra de estudio estaba compuesta por 65 niños (edades de 10 a 13 años) con diagnóstico de TA, pero sin diagnóstico clínico de trastorno de ansiedad. De acuerdo con la información de los niños y de los padres, los niños con TA mostraron significativamente más problemas de ansiedad que los niños con desarrollo típico. Basándose en los auto-informes de los niños, la intensidad de la sintomatología ansiógena era similar en los niños con TA y en aquellos con fobia social. Sin embargo, la información aportada por los padres ofrecía mayores puntuaciones en ansiedad generalizada y más síntomas obsesivocompulsivos en el grupo TA que en el grupo con fobia social. También se hallaron desacuerdos en las evaluaciones sobre las preocupaciones sociales de los niños con TA, pues los padres informaban de mayores preocupaciones sociales que las que referían los propios niños. Los autores concluyen que aunque los niños con TA pueden experimentar ansiedad significativa, pueden tener dificultades para informar sobre ella. Por esta razón, sugieren que los informes de los padres podrían ser una información más precisa y fiable.

En este sentido, en la literatura se viene recomendando el uso de múltiples informantes (por ejemplo, familia y sujeto con TEA) mediante escalas de calificación que permitan evaluar los síntomas psicopatológicos comórbidos (White et al., 2009; White y Roberson-Nay, 2009).

El DSM IV-TR (APA, 2002) subraya similitudes conductuales entre el Trastorno Obsesivo Compulsivo de la Personalidad (TOCP) y el Trastorno Obsesivo-Compulsivo -en adelante, TOC-; además, señala posibles solapamientos de los criterios diagnósticos de ambos con las conductas ritualísticas y comportamientos repetitivos propios tanto del autismo como del TA (Gillberg y Billstedt, 2000). De hecho, ya desde la concepción inicial del síndrome de "psicopatía autística" descrito por Hans Asperger (1944) se recoge una coincidencia en dicha sintomatología. En las primeras descripciones del autismo, Leo Kanner (1943) observó que varios de los niños estudiados tenían considerables problemas de ansiedad. Sugería que las características nucleares del déficit, especialmente el deseo de invarianza, las rutinas, obsesiones, el repertorio de comportamientos repetitivos, obsesivos y estereotipados, etc. (criterio $b$ para el TA y $c$ para el Trastorno Autista del actual DSM IV-TR, APA, 2002), podrían estar relacionadas con la manifestación de sintomatología depresiva y ansiosa. Esta ansiedad podría agudizar esta sintomatología, que podría actuar como estrategia autorelajante, procurando controlar la ansiedad mediante obsesiones y rituales (Gillot et al., 2001; Melfsen et al., 2006).

Charlop-Christy y Haymes (1996) evaluaron las obsesiones de un grupo de niños con TEA. En concreto, en su estudio definieron operativamente las obsesiones como las "solicitudes verbales continuas", y consideraron que las con- 
ductas obsesivas (por ejemplo, consultar mapas, lanzar una pelota, etc.), eran una forma de comportamientos estereotipados propios de los TEA. Además, resultan interesantes los resultados sobre la no universalidad de estos comportamientos obsesivos en toda la muestra de niños con TEA. Así, los autores consideran que el comportamiento obsesivo puede aparecer como parte de la sintomatología de los TEA, más que como una condición comórbida diferente. Desde este planteamiento, se propone que la depresión sería el extremo más distintivo de un continuo, mientras que la conducta obsesiva-compulsiva se hallaría en el otro, debido a su similitud con la sintomatología esencial del TEA (Matson y NebelSchwalm, 2007). Sin embargo, son necesarios más estudios descriptivos de la caracterización de las conductas o síntomas, para definir con mayor precisión si pueden presentarse en los TEA diferentes tipos de obsesiones y compulsiones, y de ser así, cuándo y con qué frecuencia.

Desde otra vertiente, se apunta una relación y solapamiento parcial de los dos trastornos (Paula-Pérez y MartosPérez, 2009). Sin embargo, se reconoce que la aplicación de la categoría diagnóstica TOC en los TEA puede no estar clara, y que son necesarios estudios adicionales que ayuden a comprender mejor las características de los pensamientos y conductas repetitivas en los TEA, y a aclarar la base subyacente de los síntomas neurobiológicos hallados (Ruta, Mugno, D’Arrigo, Vitiello y Mazzone, 2010).

Este planteamiento parece sostenido por algunas evidencias (Ruta et al., 2010). Se halla una mayor prevalencia de rasgos autistas en sujetos con TOC tanto adultos (Bejerot, 2007; Bejerot, Nylander, Lindstro" $m$, 2001) como niños (Ivarsson y Melin, 2008). Además, existen ensayos clínicos que encuentran que los tratamientos farmacológicos para el TOC pueden resultar eficaces para los pensamientos y conductas repetitivas en los TEA (por ejemplo, los Inhibidores de Recaptación de Serotonina) (Hollander et al., 2005; Lehmkuhl, Storch, Bodfish y Geffken, 2007; McDougle, Kresch y Posey, 2000). En el mismo sentido, los estudios con familiares hallan una asociación entre conductas restrictivas o repetitivas de las muestras con autismo y características del TOC en sus padres, tanto en familias con casos múltiples (Hollander, King, Delaney, Smith y Silverman, 2003) como en familias con casos únicos (Abramson et al., 2005).

Cath, Ran, Smit, van Balkom y Comijs (2008) se centraron en el estudio de los síntomas que se superponen entre los TEA y los TOC en adultos. Los sujetos exclusivamente con TOC obtuvieron mayores puntuaciones en la severidad sintomatológica de obsesiones y compulsiones. En aquéllos con TOC y TEA hallaron un nivel intermedio entre los sujetos con TOC y los controles con desarrollo típico. No se encuentran diferencias significativas en el grado de insight entre los sujetos con TOC, con y sin TEA. Otro estudio con adultos (Russell, Mataix-Cols, Anson y Murphy, 2005) compara sujetos con TEA-AF y sujetos con TOC. Hallan un patrón de obsesiones y compulsiones similar en el grupo con TEAAF y el grupo con TOC, y un 25\% de los sujetos con TEA-
AF recibieron además un diagnóstico clínico de TOC de acuerdo con los criterios de la CIE-10 (OMS, 1992).

Entre los escasos estudios centrados en investigar la presencia de rasgos obsesivos-compulsivos en niños con TEAAF, destaca el trabajo de Zandt, Prior y Kyrios (2007). En esta investigación se comparan niños con TEA-AF y con TOC en una amplia gama de conductas repetitivas. Los sujetos con TOC y con TEA-AF mostraron tasas similares de conductas y movimientos repetitivos. Sin embargo, destaca que se encuentran mayores tasas de conductas repetitivas en los niños con TOC más jóvenes que en los mayores, mientras que en el grupo con TEA-AF no se hallan diferencias según el criterio de edad. Además, el grupo con TOC mostró más frecuentes y más sofisticadas obsesiones y compulsiones que el grupo con autismo de alto funcionamiento. Ambos grupos mostraron más obsesiones y compulsiones que el grupo control con desarrollo típico.

En un reciente estudio que intenta dilucidar si los síntomas de TOC han de ser considerados comórbidos según el enfoque categorial o si se les puede considerar como un continuo de síntomas, Ruta et al. (2010) examina la presencia y características del TOC en niños y adolescentes de entre 8 y 15 años con TA $(\mathrm{n}=18)$ respecto a un grupo con TOC $(n=20)$ y otro grupo control con desarrollo típico $(n=22)$, equiparados en edad, género y CI. La gravedad de los síntomas obsesivos-compulsivos fue significativamente más alta no sólo en el grupo TOC (rango moderado de gravedad), sino también en los niños con TA. Los autores esgrimen que los síntomas obsesivos-compulsivos hallados en su muestra con TA pueden ser interpretados como un posible solapamiento entre los TEA y el TOC, de acuerdo con estudios previos (Bartz y Hollander, 2006). De nuevo este grupo explica la mayor puntuación en obsesiones y compulsiones en el grupo con TA a partir de las dificultades específicas del colectivo. Subrayan las dificultades de los niños con TA a la hora de atribuir correctamente sus creencias rígidas y rituales, debido posiblemente a un deterioro parcial de la capacidad de procesamiento e información de sus estados internos. No obstante, destacan que ninguno de los niños con TOC o TA mostraron insight adecuados, sino deficientes y empobrecidos. Esto lo relacionan con los resultados de otras investigaciones (Geller, 2006; Piacentini, Bergman, Keller y McCracken, 2003) que hallan en los niños con TOC dificultades en la percepción, procesamiento y descripción de los pensamientos y conductas repetitivas, mediatizadas por la edad y el nivel de desarrollo, así como con la similitud en el malestar clínicamente significativo entre el TOC y TEA (Cath et al., 2008) o con los datos de Ivarsson y Melin (2008), quienes tampoco encuentran relación entre el nivel de insight en su muestra de niños con TOC y la presencia de rasgos de TEA. 


\section{Hipótesis explicativas de la comorbilidad diagnóstica y/o sintomatológica de los TEA y los Trastornos de Ansiedad}

Existen estudios que han examinado los síntomas de ansiedad en niños con TEA desde un punto de vista evolutivo (e.g., Farrugia y Hudson, 2006). Lo mismo que ocurre con los niños de desarrollo típico, suele destacarse un incremento de los trastornos de ansiedad en la adolescencia (Ollendick, King y Muris, 2004). En los niños más pequeños con TEA suelen predominar las fobias específicas, mientras que durante la adolescencia aparecen en mayor medida los trastornos como el TOC y la fobia social (White et al., 2009).

Actualmente no está claro por qué la ansiedad aparece y es tan frecuente en la adolescencia de las personas con TEA. Entre las posibles explicaciones de corte biológico, destacan las investigaciones que aluden a defectos estructurales (Amaral, Bauman y Schumann, 2003) y/o neuroquímicos (Apter, Brown, Korn, y van Praag, 1991). Estas alteraciones cerebrales podrían predisponer a las personas con TEA a experimentar ansiedad. Desde esta misma perspectiva, también se especula con un posible vínculo genético entre los TEA y la ansiedad (Piven y Palmer, 1999; Smalley, McCracken y Tanguay, 1995), aunque los mecanismos concretos aún son desconocidos.

Desde una perspectiva psicosocial se destaca además que el momento de la transición de la niñez a la adolescencia en los TEA puede representar importantes retos. Para los adolescentes con TEA, la necesidad de adaptación a los nuevos contextos socialmente más complejos, los cambios de rutina en la escuela o de nivel educativo pueden llegar a ser una experiencia abrumadora (White et al., 2009). Además, los cambios físicos, hormonales, socio-emocionales, etc propios de esta etapa serán retos a sumar a las nuevas expectativas y demandas familiares, sociales y académicas (Khouzam, ElGabalawi y Priest, 2004).

Desde un enfoque bidireccional, centrado en las características patognómicas del trastorno, se conjeturan posibles efectos de mediación, que funcionarían como círculo explicativo, entre la ansiedad, sus dificultades en interacción social, la conciencia de ser diferente de los demás, especialmente durante la adolescencia, la soledad y el aislamiento social. En concreto, las propias dificultades sociales de las personas con TEA pueden desembocar en ansiedad relacionada con la mala interpretación de las reglas sociales y con sus expectativas de fracaso social (White et al., 2009; White y Roberson-Nay, 2009). Además, y especialmente en la adolescencia de las personas con mayor capacidad cognitiva, serán "muy conscientes de sus dificultades de integración social" (Attwood, 2000, p. 97), de su soledad y dificultades sociales (Attwood, 2000; Schopler y Mesibov, 1983; Tantam, 2003 White et al., 2009; White y Roberson-Nay, 2009). Esta mayor consciencia de sus dificultades sociales, puede a su vez contribuir al desarrollo de trastornos del estado de ánimo y problemas de ansiedad, depresión u hostilidad (Myles, 2003; Tantam,
2003). A su vez, esta ansiedad generada puede incrementar las conductas de evitación de situaciones sociales, las interacciones sociales, etc., contribuyendo a un mayor aislamiento, agravando por tanto más su déficit social. A todo esto hemos de añadir que, a menudo, los propios niños y adolescentes con TEA-AF informan de su deseo de tener mayores interacciones con sus compañeros (White y Roberson-Nay, 2009), y se constata experimentalmente que experimentan una mayor soledad que su grupo de iguales (Bauminger y Kasari, 2000). Sin embargo, sus dificultades sociales, especialmente con compañeros de su misma edad, hacen que esto sea difícil, lo que nos llevaría de nuevo al inicio de esta explicación circular.

Desde esta óptica psicosocial, existe una corriente explicativa que argumenta que estas mayores tasas de ansiedad social en el grupo de TA son esperables, pues los niños y adolescentes con trastornos mentales están expuestos a mayores niveles de estrés. Así, desde el modelo de vulnerabilidad-estrés, las propias características del trastorno, interactuando con los factores de vulnerabilidad-estrés y las experiencias en el medio, pueden dar como resultado el aumento de los trastornos de ansiedad tanto como comorbilidad diagnóstica como sintomatológica (Melfsen et al., 2006).

En esta línea, el estudio de seguimiento de Hutton, Goode, Murphy, Le Couteur y Rutter (2008) destaca que la aparición de trastornos comórbidos en su muestra estuvo generalmente asociada a acontecimientos vitales estresantes (por ejemplo, en los sujetos con menor nivel de funcionamiento cognitivo, un cambio de residencia, de cuidadores o en las rutinas familiares; y en los casos con mayor funcionamiento cognitivo, la pérdida de empleo o la muerte de un ser querido). En el seguimiento durante, al menos, 21 años de 135 personas con TEA $(\mathrm{CI}>30)$ hallan, mediante entrevistas a los familiares, 39 casos con un posible trastorno comórbido de nueva aparición en la adolescencia o adultez, destacando las alteraciones afectivas, y los trastorno obsesivo-compulsivos y catatonía, independientemente del funcionamiento cognitivo.

\section{Discusión y conclusiones}

Teniendo en cuenta los propósitos de este trabajo, a continuación exponemos y discutimos las posibles implicaciones derivadas de los análisis realizados:

a) Acerca del estado actual del corpus cientifico sobre comorbilidad de los TEA y los trastornos de ansiedad, hemos de destacar que existe una importante evidencia científica sobre la alta tasa de comorbilidad. Sin embargo, existen escasos estudios sobre la caracterización, los efectos específicos de la ansiedad en los adolescentes con TEA y su curso evolutivo, la epidemiología, evaluación y tratamiento en este colectivo. Sólo recientemente ha comenzado a recibir la atención que se merece, pero aún continúa siendo insuficiente (White et al., 2009). 
b) Sobre los posibles problemas metodológicos de las diferentes investigaciones, destacamos que, en general, la mayoría de los trabajos adolecen de limitaciones metodológicas. Los estudios son escasos y muy variables en cuanto al tamaño de la muestra (normalmente reducida), los rangos de edad, diagnósticos y los métodos de confirmación, etc., por lo que resulta difícil la comparación y generalización de los resultados. Todas estas limitaciones experimentales parecen fundamentadas, por un lado, por las características propias de la muestra: por ejemplo, baja prevalencia, falta de acuerdo unánime hasta el momento sobre los criterios diagnósticos para el TA (APA, 2011), etc., y por otro, por las dificultades específicas en la evaluación de la ansiedad en los TEA, que hacen que autores como White et al. (2009) planteen que puede estar infradiagnosticada. Quizás también pueda proponerse, incluso, el trabajo en entornos virtuales (como se propone por Suriá y Beléndez, 2011, mediante grupos virtuales de autoayuda).

En este sentido, no existe un acuerdo sobre el método clínico más adecuado para evaluar este tipo de sintomatología en los TEA. En población sin trastornos, se ha venido evaluando fundamentalmente mediante cuestionarios, inventarios y escalas (Martínez, Cano, Castillo, Sánchez, Ortiz y Gordillo, 1995). Sin embargo, autores como White y Roberson-Nay (2009) critican la inadecuada extrapolación de pruebas tipificadas para el uso en población sin trastornos, pues pueden subestimar ciertas manifestaciones idiosincrásicas e inusuales que se observan en este colectivo (como puede ser la exacerbación de los comportamientos repetitivos, White y Roberson-Nay, 2009). Por ejemplo, en la población con TEA el uso de instrumentos de auto-informe podrá comprometer sus capacidades de introspección, algo que se agudiza en el colectivo con discapacidad intelectual (Baron-Cohen, 1989; Capps et al., 1992; Melfsen et al., 2006; White et al., 2009; White y Roberson-Nay, 2009). Así, algunos estudios han encontrado que los resultados de los auto-informes sobre sintomatología ansiógena en jóvenes con TEA-AF son menos precisos que la información aportada por los familiares (Russell y Sofronoff, 2005), aunque también existen investigaciones en que estos datos sí correlacionan positivamente con los aportados por sus familiares ( $\mathrm{Fa}-$ rrugia y Hudson, 2006). Hasta el momento, y aunque se recomienda el uso de múltiples informantes, la mayoría de los estudios se basan exclusivamente en los informes de familiares, aunque también existen estudios con una combinación de esta información ofrecida por los familiares y por los propios afectados (por ejemplo, Bellini, 2004; Gadow, DeVincent, Pomeroy y Azizian, 2005; Lecavalier, 2006). Por otra parte, en la mayoría de los estudios no se especifica si la ansiedad evaluada se corresponde con la ansiedad estado (estado emocional transitorio de ansiedad) o con la ansiedad rasgo (tendencia individual a reac- cionar de forma ansiosa) (Spielberger, Gorsuch y Lushene, 1970). Este dato es de suma importancia para el diagnóstico diferencial y el tratamiento de los problemas de ansiedad comórbidos.

Así, es importante considerar que aunque las manifestaciones clínicas de ansiedad en los jóvenes con autismo pueden ser similares en muchos aspectos a las observadas en el desarrollo típico, existen algunos aspectos a tener en cuenta en la selección de los instrumentos, pues existe el riesgo de sesgar la evaluación: (1) la ansiedad en los TEA puede mostrar signos idiosincrásicos (por ejemplo, el aumento de actividad quinética, aumento de conductas repetitivas o de la intensidad de sus intereses restringidos, White y Roberson-Nay, 2009); (2) el propio trastorno a menudo implica patognómicamente la manifestación de síntomas como miedos específicos, hiperactividad, obsesiones, estereotipias, etc., que hace complicado diferenciarlos de síntomas afectivos o ansiosos, que pueden pasar desapercibidos entre la sintomatología propia del síndrome (ver más arriba TOC, Paula-Pérez y Martos-Pérez, 2009); (3) la ansiedad puede complicarse al interactuar con las características propias del TEA al asociarse, por ejemplo, con las deficiencias en la simbolización (Wing y Gould, 1979); y (4) los adolescentes con TEA pueden carecer del conocimiento de sus sentimientos y estados internos (Baron-Cohen, 1989), así como de sus pensamientos ansiógenos, y a pesar de ser capaz de describir los signos evidentes de ansiedad (por ejemplo, conductas de evitación), no asociarlos a la ansiedad y por tanto no informar de manera precisa sobre sus síntomas.

c) A propósito del amplio debate sobre si el TEA puede estar separado clínicamente del TOC y las diferentes explicaciones sobre la comorbilidad (diagnóstica o sintomatológica), así como la aportación final reflexiva de este trabajo acorde con los resultados obtenidos a partir de estos análisis, destacamos que los comportamientos o actos mentales repetitivos que la persona con TOC se ve obligada a realizar en respuesta a una obsesión o reglas estrictas con el objetivo de prevenir o reducir el malestar (criterio a para el TOC del actual DSM IV-TR, APA, 2002), en principio parecen cumplir el criterio de inflexibilidad conductual y mental que encontramos en los TEA (criterio $b$ para el TA y c para el Trastorno Autista, APA, 2002). Además, aunque la fenomenología clínica de los síntomas obsesivos-compulsivos parece similar en las personas con TOC y en los individuos con TEA, éstos últimos podrían tener poca conciencia (insight) del carácter excesivo e irracional de la naturaleza de sus pensamientos y conductas repetitivas (egodistonicidad, Ruta et al., 2010), lo que es preciso para la configuración diagnóstica del TOC (APA, 2002). Sin embargo es importante recordar que, si bien este malestar clínicamente significativo generado por esta sintomatología parece ser una característica típica de los TOC 
en la edad adulta, este criterio diagnóstico del DSM-IVTR (APA, 2002) no se aplica a los niños con TOC, quienes pueden carecer de suficiente conciencia cognitiva para poder hacer estas reflexiones (Geller, 2006). En este sentido, la dificultad de las personas con autismo con la capacidad de introspección está bien documentada (BaronCohen, 1989; Capps, Yirmiya y Sigman, 1992; Melfsen et al., 2006; White et al., 2009; White y Roberson-Nay, 2009), y puede afectar a su capacidad para informar de la sintomatología relativa al cuadro: "los individuos autistas muestran un deterioro en la capacidad para procesar y hablar de su propio estado mental interno" (Baron-Cohen, 1989, p. 193). No obstante, para el diagnóstico diferencial entre la ansiedad y las características propias de los TEA deberíamos conocer con exactitud si, aunque la respuesta conductual (e.g. lavarse repetidamente las manos) es similar, existe un sustrato causal diferente (e.g. con la finalidad de matar los gérmenes). Para ello precisaríamos conocer la experiencia subjetiva de los propios afectados.

Este solapamiento procurará ser resuelto en el DSM $\mathrm{V}$, facilitando el diagnóstico diferencial respecto a la futura categoría de Espectro de Trastornos del Autismo al excluir los "patrones repetitivos del comportamiento en los TEA" (criterio $d$ para el diagnóstico de Trastorno del Espectro de Ansiedad y Obsesivo Compulsivo del futuro DSM V, APA, 2011).

En cuanto a la posible etiología o factores de riesgo, destacamos los estudios desde lo que hemos denominado la "vertiente psicosocial", sobre la influencia de factores biológicos (genéticos, Piven y Palmer, 1999) y psicosociales como la edad (adolescencia) y el nivel cognitivo (mayor funcionamiento), así como las propias dificultades asociadas a los TEA -déficit en habilidades sociales- (Gadow et al., 2005). Así, y a la vista de los resultados, nuestra propuesta, siguiendo a Caron y Rutter (1991) y su análisis sobre la comorbilidad, es que la co-ocurrencia de los trastornos del espectro autista y del espectro de ansiedad estaría relacionada, por un lado, con que las propias dificultades del TA pueden representar una manifestación temprana de la ansiedad, que en la adolescencia se agravaría al ser conscientes de sus dificultades; y que la comorbilidad puede ser debida al solapamiento de criterios diagnósticos.

En conclusión, dentro del colectivo de los TEA encontramos un grupo de alto riesgo para los trastornos de ansiedad. La adolescencia y el mayor nivel cognoscitivo supondrán factores de riesgo a tener en cuenta, que mediarán con la influencia de las dificultades propias del trastorno, especialmente las comunicativas y socioemociona- les. Sin embargo, aún son escasos los trabajos que tratan de identificar posibles factores causales.

\section{Líneas futuras de investigación}

Aún son precisos más estudios para la caracterización de la ansiedad en los TEA que profundicen sobre las manifestaciones sintomatológicas, los efectos específicos en este colectivo, su evolución, tasas de epidemiología y prevalencia (con muestras más numerosas y con uniformidad de diagnósticos), evaluación y tratamiento en este colectivo. Destaca además la llamativa escasez de estudios de investigación en cualquiera de estas áreas en países de lengua hispana. Puesto que la co-ocurrencia de estos trastornos merece atención e intervención específica, existe una necesidad urgente de desarrollar métodos eficaces para evaluar y tratar la ansiedad de los niños y adolescentes con TEA.

En cuanto a la evaluación, será preciso un acuerdo sobre cómo evaluar este tipo de sintomatología en los TEA, y el diseño de estrategias e instrumentos específicos que ayuden a la evaluación de esta sintomatología comórbida. Esta evaluación deberá tener en cuenta la posible manifestación idiosincrásica de la sintomatología ansiógena, el posible solapamiento entre las obsesiones y compulsiones y las características de los TEA, que además puede complicarse al interactuar con las características propias del cuadro principal, y las dificultades de estos sujetos en la comunicación en general y en la comunicación sobre sus propias vivencias y sintomatología en particular.

Por lo que respecta a la intervención, son precisos estudios que puedan establecer las relaciones entre los factores de riesgo de manera empírica. De esta manera, podremos comprobar si existe una disminución en la ansiedad cuando logran mejorar sus dificultades sociales y comunicativas con intervenciones que tengan en cuenta que la persona con TA es intensamente consciente de su desconexión social (Atwood, 2000).

Todos estos avances se hacen necesarios para poder prevenir, identificar y tratar una posible comorbilidad diagnóstica o sintomatológica, pues en muchos casos, y bajo el paraguas sintomatológico del TA (APA, 2002), puede esconderse un trastorno de ansiedad que ha de recibir intervención específica y que además puede agravar la propia sintomatología patognómica del TA.

Agradecimientos.- La realización de este trabajo ha sido posible gracias a la Subvención concedida por la Consejería de Educación de la Junta de Castilla y León a Myriam De la Iglesia (Resolución ORDEN EDU/1278/2011, de 7 de octubre; BOCyL 18-10-2011). 


\section{Referencias}

Abramson, R.K., Ravan, S.A., Wright, H.H., Wieduwilt, K., Wolpert, C.M., Donnelly, S.A., Pericak-Vance, M.A. y Cuccaro, M.L. (2005). The relationship between restrictive and repetitive behaviors in individuals with autism and obsessive compulsive symptoms in parents. Child Psychiatry and Human Development, 36, 155-165.

Amaral, D.G., Bauman, M.D. y Schumann, C.M. (2003). The amygdale and autism: Implications from non-human primate studies. Genes Brain and Behavior, 2, 295-302.

Asociación Americana de Psiquiatría. (2002). Manual Diagnóstico y Estadístico de los Trastornos Mentales. Texto Revisado. DSM-IV-TR. Barcelona: Masson.

Asociación Americana de Psiquiatría. (2011, 28 febrero). DSM-5 Development. Recuperado 28 febrero, 2011, de http://www.dsm5.org/Pages/Default.aspx

Apter, A., Brown, S.L., Korn, M.L. y van Praag, H.M. (1991). Psychiatric disorders of childhood: The role of serotonin. En S.L. Brown y H.M. van Praag (Eds.), Clinical and experimental psychiatry monograph: No. 4. The role of serotonin in psychiatric disorders (pp. 215-238). Philadelphia: Brunner/Mazel.

Asperger, H. (1944). De "Autistischen Psychopathen" im Kindesalter. Archiv für Psychiatrie und Nervenkrankheiten, 117, 76-136. Trad. Inglés por U. Frith (1991). Translations and annotation of "autistic psychopathy" in childhood, by H. Asperger. En U. Frith (1991). Autism and Asperger syndrome (pp. 37-92).Cambridge: Cambridge University Press.

Attwood, T. (2000). Strategies for improving the social integration of children with Asperger syndrome. Autism, 4, 85-100.

Baron-Cohen, S. (1989). Perceptual role taking and protodeclarative pointing in autism. British Journal of Developmental Psychology, 7, 113127.

Bartz, J.A. y Hollander, E. (2006) Is obsessive-compulsive disorder an anxiety disorder? Progress in neuro-psychopharmacology and biological psychiatry, 30, 338-352.

Bauminger, N. y Kasari, C. (2000). Loneliness and friendship in highfunctioning children with autism. Child Development, 71, 447-456.

Bejerot, S. (2007) An autistic dimension: a proposed subtype of obsessive-compulsive disorder. Autism, 11, 101-110.

Bejerot, S., Nylander, L. y Lindstro"m, E. (2001) Autistic traits in obsessive-compulsive disorder. Nordic Journal of Psychiatry, 55, 169176.

Bellini, S. (2004). Social skill deficits and anxiety in high-functioning adolescents with autism spectrum disorders. Focus on Autism and Other Developmental Disabilities, 19, 78-86.

Bishop, D.V.M. (1989). Autism, Asperger Syndrome and Semantic Pragmatic Disorder - Where are the boundaries?. British Journal of Disorders of Communication, 24, 107-121.

Brereton, A., Tonge, B.J. y Einfeld, S.L. (2006). Psychopathology in children and adolescents with autism compared to young people with intellectual disability. Journal of Autism and Developmental Disorders, 36, 863-870.

Capps, L., Yirmiya, N., Sigman, M. (1992) Understanding of simple and complex emotions in non-retarded children with autism. Journal of Child Psychology and Psychiatry, 33, 1169-1182.

Caron, C. y Rutter, M. (1991). Comorbidity in child psychopathology: Concepts, issues, and research strategies. Journal of Child Psychology and Psychiatry, 32, 1063-1080.

Cath, D.C., Ran, N., Smit, J.H., van Balkom, A.J. y Comijs, H.C. (2008) Symptom overlap between autism spectrum disorder, generalized social anxiety disorder and obsessive-compulsive disorder in adults: a preliminary case-controlled study. Psychopathology, 41, 101-110.

Charlop-Christy, M.H. y Haymes, L.K. (1996). Using obsessions as reinforcers with and without mild reduction procedures to decrease inappropriate behaviors of children with autism. Journal of Autism and Developmental Disorders, 26, 527-546.
Damián, M. y Hernández, A. (1997). Estudio longitudinal de una niña autista. Anales de psicología, 13 (2), 143-154.

de Bruin, E.I., Ferdinand, R.F., Meester, S., de Nijs, P.F.A. y Verheij, F. (2006). High rates of psychiatric co-morbidity in PDD-NOS. Journal of Autism and Developmental Disorders, 37, 877-886.

Evans, D.W., Canavera, K., Kleinpeter, F.L., Maccubbin, E. y Taga, K. (2005). The fears, phobias and anxieties of children with autism spectrum disorders and Down syndrome: Comparisons with developmentally and chronologically age matched children. Child Psychiatry and Human Development, 36, 3-26.

Farrugia, S. y Hudson, J. (2006). Anxiety in adolescents with Asperger syndrome: Negative thoughts, behavioral problems, and life interference. Focus on Autism and Other Developmental Disabilities, 21, 2535.

Gadow, K.D, DeVincent, C.J., Pomeroy, J. y Azizian, A. (2004). Psychiatric symptoms in preschool children with PDD and clinic and comparison samples. Journal of Autism and Developmental Disorders, 34, 379-393.

Gadow, K.D., DeVincent, C.J., Pomeroy, J. y Azizian, A. (2005). Comparison of DSM-IV symptoms in elementary school-age children with PDD versus clinic and community samples. Autism, 9, 392415.

Geller, D.A. (2006) Obsessive-compulsive and spectrum disorders in children and adolescents. Review. Psychiatric Clinics of North America, 29, 353-370.

Ghaziuddin, M. (2002). Asperger syndrome: Associated psychiatric and medical conditions. Focus on Autism and Other Developmental Disabilities, 17, 138-144.

Ghaziuddin, M., Ghaziuddin, N. y Greden, J. (2002). Depression in persons with autism: implications for research and clinical care. Journal of Autism and Developmental Disorders, 32, 299-306.

Gillberg, C. y Billstedt, E. (2000). Autism and Asperger syndrome: coexistence with other clinical disorders. Acta Psychiatrica Scandinavica, 102, 321-330.

Gillot, A., Furniss, F. y Walter, A. (2001) Anxiety in high-functioning children with autism. Autism, 5, 277-286.

Green, J., Gilchrist, A., Di Burton, y Cox, A. (2000). Social and psychiatric functioning in adolescents with Asperger Syndrome compared with conduct disorder. Journal of Autism and Developmental Disorders, 30, 279-293

Holguin, J.A. (2003). El autismo de etiología desconocida. Revista de Neurología, 37, 259-266.

Hollander E, King A, Delaney K, Smith CJ. y Silverman, J.M. (2003). Obsessive-compulsive behaviors in parents of multiplex autism families. Psychiatry Research, 117, 11-16.

Hollander, E., Phillips, A., Chaplin, W., Zagursky, K., Novotny, S., Wasserman, S. y Iyengar, R. (2005) A placebo controlled crossover trial of liquid fluoxetine on repetitive behaviors in childhood and adolescent autism. Neuropsychopharmacology, 30, 582-589.

Hutton, J., Goode, S., Murphy, M., Le Couteur, A. y Rutter, M. (2008). New-onset psychiatric disorders in individual with autism. Autism, 12, 373-90.

Ivarsson, T, y Melin, K. (2008) Autism spectrum traits in children and adolescents with obsessive-compulsive disorder (OCD). Journal of Anxiety Disorders, 22, 969-978.

Kanner, L. (1943). Autistic disturbances of affective contact. Nervous Child, 2, 217-250. (Trad. Cast. por T. Sanz, en Siglo Cero, 1993, $n^{\circ}$ 149).

Khouzam, H.R., El-Gabalawi, F. y Priest, F. (2004). Asperger's disorder: A review of its diagnosis and treatment. Comprehensive Psychiatry, 45, 183-191.

Kim, J.A., Szatmari, P., Bryson, S.E., Streiner, D.L. y Wilson, F.J. (2000). The prevalence of anxiety and mood problems among children with autism and Asperger syndrome. Autism, 4, 117-132. 
Klin, A. y Volkmar, F.R. (1997). Asperger`s Syndrome. En D.J. Cohen y F.R. Volkmar (Eds.), Handbook of autism and pervasive developmental disorders, 2nd Edit., (pp. 94-122). Nueva York: John Wiley and Sons.

Lecavalier, L. (2006). Behavioral and emotional problems in young people with pervasive developmental disorders: Relative prevalence, effects of subject characteristics, and empirical classification. Journal of Autism and Developmental Disorders, 36, 1101-1114.

Lehmkuhl, H.D., Storch, E.A., Bodfish, J.W. y Geffken, G.R. (2007) Brief report: exposure and response prevention for obsessive compulsive disorder in a 12-year-old with autism. Journal of Autism and Developmental Disorders, 38, 977-981.

Leyfer, O.T., Folstein, S.E., Bacalman, S., Davis, N.O., Dinh, E., Morgan, J., Tager-Flusberg, H. y Lainhart, J.E. (2006). Comorbid psychiatric disorders in children with autism: Interview development and rates of disorders. Journal of Autism and Developmental Disorders, 36, 849-861.

López, C; Alcántara, M.V; Fernández, V; Castro, M. y López, J.A. (2010). Características y prevalencia de los problemas de ansiedad, depresión y quejas somáticas en una muestra clínica infantil de 8 a 12 años, mediante el CBCL (Child Behavior Checklist). Anales de psicologia, 26, 325-334.

Martínez , F; Cano, A; Castillo, J.C; Sánchez, J.A; Ortiz, B. y Gordillo, E. (1995). Una Escala Reducida de Ansiedad basada en el Inventario de Situaciones y Respuestas de Ansiedad (I.S.R.A.): Un estudio exploratorio. Anales de psicología, 11, 97-104.

Matson, J.L. y Nebel-Schwalm, M.S. (2007). Comorbid psychopathology with autism spectrum disorder in children: An overview. Research in Developmental Disabilities, 28, 341-352.

Mazefsky C.A., Folstein, S.E. y Lainhart, J.E. (2008). Overrepresentation of mood and anxiety disorders in adults with autism and their first-degree relatives: what does it mean?. Autism research, 1, 193197.

McDougle, C.J., Kresch, L.E. y Posey, D.J. (2000) Repetitive thoughts and behaviour in pervasive developmental disorders: treatment with serotonin reuptake inhibitors. Journal of Autism and Developmental Disorders, 30, 427-435.

Melfsen, S., Walitza, S. y Warnke, A. (2006). The extent of social anxiety in combination with mental disorders. European Child and Adolescent Psychiatry, 15, 111-117.

Myles, B. (2003). Behavioral forms of stress management for individuals with Asperger syndrome. Child and Adolescent Psychiatric Clinics of North America, 12, 123-141.

Ollendick, T.H., King, N.J. y Muris, P. (2004). Phobias in children and adolescents. En: M. Maj, H.S. Akiskal, J.J. López-Ibor, A. Ocaza (Eds.) Phobias (pp. 245-279). London: John Wiley and Sons, Inc.

Organización Mundial de la Salud (1992). Clasificación internacional de enfermedades (CIE-10). Madrid: Meditor.

Organización Mundial de la Salud (2011, 28 febrero). ICD Update and Revision Platform. Recuperado 28 febrero, 2011, de https://extranet.who.int/icdrevision/icd10p.aspx

Orgilés, M; Espada, J.P; García, J.M; Méndez, X. e Hidalgo, M.D. (2011). Most feared situations related to separation anxiety and characteristics by age and gender in late childhood. Anales de psicología, 27, 80-85.

Paula-Pérez, I. y Martos-Pérez, J. (2009). Síndrome de Asperger y autismo de alto funcionamiento: Comorbilidad con trastornos de ansiedad y del estado de ánimo. Revista de Neurología, 48, 31-34.

Piacentini, J., Bergman, R.L., Keller, M. y McCracken, J. (2003) Functional impairment in children and adolescents with obsessivecompulsive disorder. Journal of Child and Adolescent Psychopharmacology, 13, 61-69.

Piven, J. y Palmer, P. (1999). Psychiatric disorder and the broad autism phenotype: Evidence from a family study of multipleincidence autism families. The American Journal of Psychiatry, 156, 557-563.
Russell, A.J., Mataix-Cols, D., Anson, M. y Murphy, D.G. (2005) Obsessions and compulsions in Asperger syndrome and high functioning autism. The British Journal of Psychiatry, 186, 525-528.

Russell, E. y Sofronoff, K. (2005). Anxiety and social worries in children with Asperger syndrome. The Australian and New Zealand Journal of Psychiatry, 39, 633-638.

Ruta, D., Mugno, D., D’Arrigo, V.G., Vitiello, B. y Mazzone, L. (2010). Obsessive-compulsive traits in children and adolescents with Asperger syndrome. European child and adolescent psychiatry, 91, 17-24.

Rutter, M., Bailey, A., Bolton, P. y Le Couteur, A. (1994). Autism and known medical conditions: myth and substance. Journal of Child Psychology and Psychiatry, 35, 311-322.

Salavera , C., Puyuelo, M, Tricás, J.M. y Lucha, O.R. (2010). Comorbilidad de trastornos de personalidad: estudio en personas sin hogar. Universitas Psychologica, 9, 457-467.

Sanz, J. (1993). Distinguiendo ansiedad y depresión: Revisión de la hipótesis de la especificidad de contenido de Beck. Anales de psicología, 9, 133-170.

Seguí, J.D; Ortiz, M. y De Diego, Y. (2008). Factores asociados al estrés del cuidador primario de niños con autismo: Sobrecarga, psicopatología y estado de salud. Anales de psicología, 24, 100-105.

Schopler, E. y Mesibov, G. (1983). Autism in adolescents and adults. New York: Plenum Press.

Simonoff, E., Pickles, A., Charman, T., Chandler, S., Loucas, T. y Baird G. (2008). Psychiatric disorders in children with autism spectrum disorders: prevalence, comorbidity, and associated factors in a population-derived sample. Journal of the American Academy of Child and Adolescent Psychiatry, 47, 921-929.

Smalley, S., McCracken, J. y Tanguay, P. (1995). Autism, affective disorders, and social phobia. American Journal of Medical Genetics, 60, 19-26.

Spielberger, C.D., Gorsuch, R.L. y Lushene, R.E. (1970). Manual for the State-Trait Anxiety Inventory, Palo Alto: Consulting Psychologist Press. (Adaptación española STAIC, Inventario de Ansiedad Estado Rasgo. Madrid: TEA; 1988).

Sterling, L., Dawson, G., Estes, A. y Greenson, J. (2008). Characteristics Associated with Presence of Depressive Symptoms in Adults with Autism Spectrum Disorder. Journal of Autism and Developmental Disorders, 38, 1011-1018.

Stewart, M.E., Barnard, L., Pearson, J., Hasan, R. y O’Brien, G. (2006). Presentation of depression in autism and Asperger's syndrome: a review. Autism, 10, 103-116.

Sukhodolsky, D.G., Scahill, L., Gadow, K.D., Arnold, L.E., Aman, M.G., McDougle, C.J., , McCracken JT, Tierney E, Williams White S, Lecavalier L. y Vitiello B. (2008). Parent-rated anxiety symptoms in children with pervasive developmental disorders: Frequency and association with core autism symptoms and cognitive functioning. Journal of Abnormal Child Psychology, 36, 117-128.

Suriá, R. y Beléndez, M. (2011). Grupos de apoyo virtuales dedicados a problemas de salud: estudio de su tipología y análisis de su representatividad. Anales de Psicología, 27, 210-220.

Sverd, J. (2003). Psychiatric disorders in individuals with pervasive developmental disorder. Journal of Psychiatric Practice, 9, 111-127.

Tantam, D.J.H. (2000). Psychological disorder in adolescents and adults with Asperger syndrome. Autism, 4, 47-62.

Tantam, D.J.H. (2003). The challenge of adolescents and adults with asperger syndrome. Child and Adolescent Psychiatric Clinics of North America, 12, 143-163.

Volkmar F.R. y Klin, A. (2000). Diagnostic issues in Asperger syndrome. En A. Klin, F.R. Volkmar y S.S. Sparrow (Eds.), Asperger syndrome (pp. 25-71). Nueva York: The Guilford Press.

Weisbrot, D.M., Gadow, K.D., DeVincent, C.J. y Pomeroy, J. (2005). The presentation of anxiety in children with pervasive developmental disorders. Journal of Child and Adolescent Psychopharmacology, 15, 477-496. 
White, S.W. y Roberson-Nay, R. (2009). Anxiety, social deficits, and loneliness in youth with Autism Spectrum Disorders. Journal of $A u$ tism and Developmental Disorders, 39, 1006-1013.

White, S.W., Oswald, D., Ollendick, T. y Scahill, L. (2009). Anxiety in Children and Adolescents with Autism Spectrum Disorders. Clinical Psychology Review, 29, 216-229.

Wing, L. y Gould, J. (1979). Severe impairments of social interaction and associated abnormalities in children: Epidemiology and classification. Journal of Autism and Developmental Disorders, 9, 11-29.

Wing, L. (1981). Asperger's syndrome: A clinical account. Psychological Medicine, 11, 115-129.

Wolff, S. (1998). Schizoid personality in childhood: The links with Asperger syndrome, schizophrenia spectrum disorders, and elective mutism. En E. Schopler, G. B. Mesibov y L. J. Kunce (Eds.), Asperger syndrome or High-Functioning Autism? (pp. 123-142). Nueva York: Plenum.

Wolff, S. (2000). Schizoid personality in childhood and Asperger syndrome. En A. Klin, F.R. Volkmar y S.S. Sparrow (Eds.), Asperger syndrome (pp. 278-305). Nueva York: The Guilford Press.

Zandt, F., Prior, M. y Kyrios, M. (2007) Repetitive behaviour in children with high functioning autism and obsessive compulsive disorder. Journal of Autism and Developmental Disorders, 37, 251-259.

(Artículo recibido: 05-04-2011, revisado: 16-02-2012, aceptado: 19-02-2012) 